\title{
Partnering to promote apoptosis
}

Many anticancer agents function primarily through inducing apoptosis. However, because of the pleiotropic nature of cancers and the compensatory cellular mechanisms involved, the effectiveness of monotherapies may be limited. Two recent papers highlight how combining novel agents that target apoptotic pathways with other approved drugs can enhance tumour-cell apoptosis.

In the first study, Kung and colleagues investigated whether resistance to the receptor tyrosine kinase inhibitor imatinib could be overcome by targeting inhibitor of apoptosis proteins (IAPs), which can block the activity of caspases that are the final effectors of apoptosis. Although a small-molecule IAP inhibitor, LBW242, had no activity in glioma cells on its own, combination with imatinib, which inhibits plateletderived growth factor receptor (PDGFR), increased sensitization to the apoptotic effects of imatinib.

The authors then established how the synergistic induction of apoptosis is achieved. It was found that imatinib activates the intrinsic apoptosis pathway and mitochondrial outer membrane permeabilization, but caspase 3 and caspase 7 are not activated unless IAPs are simultaneously blocked with LBW242. This occurred independently of the status of the anti-apoptotic AKT pathway. When the authors determined the effect of imatinib on pro- and anti-apoptotic mediators, imatinib resulted in a reduction in the expression of NOL3, which encodes the anti-apoptotic protein ARC. NOL3 expression was associated with glioma progression in patients, and in glioma cells, increased expression of NOL3 led to decreased apoptosis.

In a mouse xenograft glioma model, animals treated with both a PDGFR inhibitor and LBW242 had complete cessation of tumour growth, whereas either agent alone had little effect. In a second glioma stem-cell-enriched model, 3 out of 7 mice treated in the combination group remained disease-free at 125 days follow-up, with no evidence of toxicity.

In the second study, Johnstone and colleagues investigated the combination of the histone deacetylase inhibitor vorinostat and an activator of TRAIL (tumour necrosis factorrelated apoptosis-inducing ligand), both of which have the ability to induce tumour-cell-selective apoptosis.

In mouse mammary and renal carcinoma cells, the combination of MD5-1 (a mouse death receptor 5 (DR5)-specific antibody that activates TRAIL receptors) and vorinostat induced synergistic apoptosis. In a mouse tumour model that mimics human breast cancer, either vorinostat or MD5-1 alone had little effect on the growth of established carcinomas. By contrast, the combination of MD5-1 and vorinostat caused complete tumour regression in $24 \%$ of tumours and suppressed the growth of the other tumours without overt toxicity.

Next, the authors investigated the mechanisms behind the synergy in vitro and in vivo. Rather than being due to the simultaneous activation of extrinsic and intrinsic apoptotic

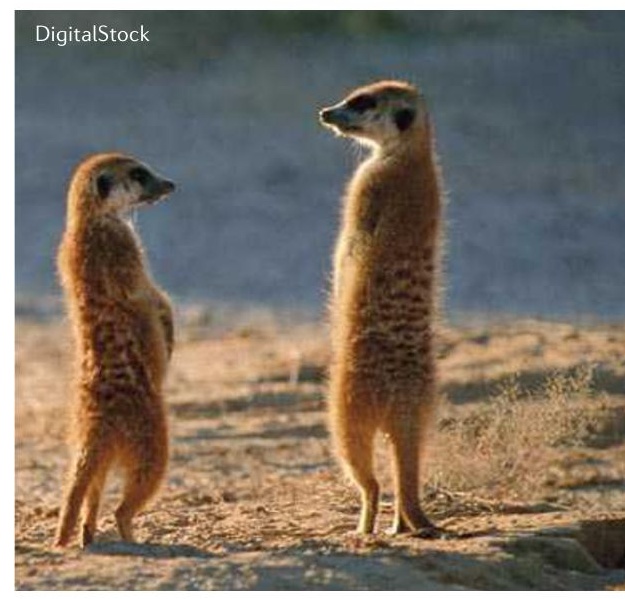

pathways, the synergistic therapeutic activities of combination therapy were mediated by the extrinsic (death-receptor mediated) apoptotic pathway. Moreover, it was found that vorinostat sensitized tumour cells to DR5-mediated apoptosis by decreasing the levels of c-FLIP - a negative regulator of caspase $8-$ and thereby relieving negative regulation of the death-receptor pathway.

These two studies highlight the therapeutic potential of targeting novel mediators in apoptotic pathways in combination with other anticancer drugs. A key issue for the future will be selecting patients that might be most likely to respond to particular combinations.

Charlotte Harrison

ORIGINAL RESEARCH PAPERS Ziegler, D. S. et al. Resistance of human glioblastoma multiforme cells to growth factor inhibitors is overcome by blockade of inhibitor of apoptosis proteins. J. Clin. Invest. 118, 3109-3122 (2008)| Frew, A. J. et al. Combination therapy of established cancer using a histone deacetylase inhibitor and a TRAIL receptor agonist. Proc. Natl Acad. Sci. USA 105, 11317-11322 (2008) 